\title{
Prólogo \\ Expansión de las fronteras internas de América Latina: El papel de Brasil en la integración regional
}

\author{
Fernando Marcelo de la Cuadra
}

Email: fmdelacuadra@gmail.com

En el marco de las transformaciones que están ocurriendo en escala global, Brasil se ha venido posicionando como una potencia a nivel regional. Este nuevo protagonismo que ha adquirido el "gigante del Sur" se debe en gran medida a la disminución de la presencia de Estados Unidos en la región, fenómeno se hizo evidente después del fracaso de su iniciativa para crear un Área de Libre Comercio para las Américas (ALCA), la cual fue definitivamente sepultada en la Cumbre de las Américas realizada en Mar del Plata en el año 2005.

De hecho, la disminución de la presencia de Estados Unidos en la región, ya venía siendo advertida por diversos analistas y desde diversas perspectivas de las relaciones internacionales. Este fenómeno viene acompañado de la crisis del hegemon que ellos representan. Para algunos autores - Hobsbawn, Wallerstein, Arrighi, Hopkins, Davis, Chomsky - dicha decadencia ya se produce lenta e inexorablemente desde los acontecimientos de mayo de 1968, la que además se habría acelerado a un ritmo vertiginoso en la última década, especialmente con posterioridad a los atentados del 11 septiembre de 2001. En América Latina, la decadencia del poder estadunidense se ha venido constatando desde antes del revés experimentado por dicha nación en la Cumbre del 2005. En efecto, la emergencia de un número significativo de coaliciones de izquierda o socialistas, moderadas o fundacionales ${ }^{1}$ ha marcado con diferentes grados e intensidades el distanciamiento de los gobiernos latinoamericanos regionales hacia las directrices y políticas emanadas desde el Departamento de Estado. A pesar de que Estados Unidos ha tratado de compensar este fracaso por medio de la firma de acuerdos bilaterales del tipo TLC, la percepción global es que Washington ha perdido un importante segmento de su influencia en el espacio regional. ${ }^{2}$

Por lo tanto, aprovechando este patente vacío del hegemon norteamericano, Brasil ha encontrado una excelente brecha para cautelar por sus intereses en el hemisferio. En este sentido, en el plano diplomático y comercial, Brasil ha sido una pieza fundamental en los esfuerzos para lograr una mayor integración regional desde su papel protagónico en la creación del MERCOSUR, inclusive con todo lo lento y complicado que ha sido el proceso de integración en esta sub-zona hemisférica. ${ }^{3}$ La formación de la Unión 
de Naciones Sudamericanas (UNASUR) significó la concreción de una virada estratégica en las relaciones bilaterales, apuntando ahora a un fortalecimiento de su posición en toda la región sur del continente. Según algunos analistas, la creación de UNASUR representó para Brasil la culminación exitosa de una concepción en que la integración regional sería funcional para su mejor desempeño como un player global, que le permitiría dividir los espacios de influencia geopolíticos y económicos con Estados Unidos. En dicho contexto, esta integración debía servirle además a Brasil en el objetivo de aumentar su poder y su autonomía en la estrategia más amplia de inserción en la economía global y de proyección en el sistema internacional. ${ }^{4}$

Un proyecto importante vinculado a UNASUR consiste precisamente en la Iniciativa para la Integración de la Infra-estructura Regional Sudamericana (IIRSA) que definió como prioritario la construcción de una red de carreteras y vías entre las diversas sub-regiones existentes en el hemisferio. El IIRSA es una propuesta que surgió con el auspicio del Banco Interamericano de Desarrollo y la Corporación Andina de Fomento (CAF) con el propósito de reordenar y reorganizar los territorios de América del Sur en corredores productivos que optimizarían los flujos de comercio y exportación. Para Brasil dicha inter-conectividad, tanto por recorridos fluviales como por carreteras, es de gran importancia, especialmente en lo que respecta a la disminución de los trayectos entre las regiones productivas y los puertos de embarque de mercaderías. Especialmente significativa para el país es la exportación de soja para el mercado Chino a través del Océano Pacifico, por eso el país ha apoyado calurosamente la creación de un corredor bioceánico que le permita tener salida hacia los puertos ubicados en el litoral occidental del continente, preferentemente en el puerto de Ilo en el sur del Perú y los de Arica e Iquique en el norte de Chile. Para ello, el gobierno de Brasil se comprometió a financiar la mayor parte de la construcción de una carretera uniría la región de Mato Grosso do Sul (principal productora de soja) con dichos puertos, atravesando el territorio boliviano y peruano. La carretera que atraviesa el territorio boliviano desde Puerto Suarez en la frontera con Mato Grosso do Sul (Corumbá) hasta la divisa con Chile, estaba siendo financiada en su mayor parte por el Estado brasileño a través del BNDES quien aportaría el 80 por ciento (US\$ 332 millones) del valor total de la obra calculada en 420 millones de dólares. La empresa que se adjudicó la licitación para la construcción de la carretera era la brasileña OAS. ${ }^{5}$

En su trazado original la carretera se proponía integrar el departamento de Cochabamba en la parte central del país con la región oriental del Beni, cruzando en su recorrido el Territorio Indígena y Parque Nacional Isiboro Sécure (TIPNIS), considerada un “área intangible” por la Constitución boliviana. Por este motivo, las comunidades indígenas que habitan el TIPNIS han realizado una serie de movilizaciones y marchas con la finalidad de alterar el trazado original que vulnera su hábitat e infringe explícitamente la Resolución 169 de la OIT que establece que ninguna actividad o proyecto productivo, extractivista o energético puede ser realizado en determina- 
do territorio sin consultar antes a las comunidades que allí habitan. Los conflictos surgidos en torno a la construcción de la carretera llevaron al gobierno de Morales a reconsiderar el inicio de los trabajos de construcción y reconocer efectivamente al TIPNIS como una zona intangible según el precepto constitucional.

Junto con ello, el gobierno decidió realizar una consulta entre las 69 comunidades que habitan el territorio, cuyo resultado favorable a la construcción de la carretera ha sido contestado por organizaciones de la Iglesia y de derechos humanos. ${ }^{6}$ Aún más, en octubre de 2012 -antes de conocerse el resultado de dicha consulta- la Empresa Boliviana de Construcción (EBC) y la Asociación de Mantenimiento Vial (AMVI) habían iniciado los trabajos del tramo I del proyecto entre Villa Tunari e Isinuta en el Departamento de Cochabamba. ${ }^{7}$

Brasil también ha tenido un importante papel en la creación de la Alianza Bolivariana para los Pueblos de las Américas (ALBA), la cual surgió con la finalidad de transformarse en una alternativa soberana al ALCA. En el contexto del ALBA, los países firmantes concibieron la posibilidad de fundar un banco que fuera una alternativa a los designios de las instituciones surgidas de Bretton Woods (Fondo Monetario Internacional y Banco Mundial), para lo cual se fundó el Banco del Sur, el cual tendría aportes significativos de Brasil junto con Venezuela. La posibilidad de tener un Banco de Fomento y Desarrollo en la región se ha frustrado, en parte por la crisis que atraviesa Venezuela y además porque Brasil ha privilegiado al Banco Nacional de Desarrollo Económico y Social (BNDES) como principal agente para el otorgamiento de préstamos a los gobiernos y empresas que actúan en la región.

Este enorme potencial genera entre determinados círculos políticos, diplomáticos y militares la convicción de que Brasil tiene que ocupar necesariamente, casi que por “destino manifiesto”, un papel trascendental en la escena internacional. De hecho, Brasil ha tenido en la última década una política exterior activa, impulsando o forjando diversas iniciativas en el plano latinoamericano y mundial, tales como creación junto con India, Alemania y Japón de un Grupo destinado a promover una reforma del Consejo de Seguridad de Naciones Unidas. ${ }^{8}$ En 2006 Brasil igualmente fue un actor fundamental en la convocatoria de un encuentro entre los países africanos y América del Sur. ${ }^{9}$ En 2010, Brasil estuvo al frente de la organización para la primera cumbre América Latina y Caribe sin la presencia de Estados Unidos, la cual permitió la creación de la Comunidad de Estados Latinoamericanos y Caribeños (CELAC).

En el contexto internacional Brasil se destaca como la séptima economía mundial y se estima que en 2014 tendrá un PIB anual de 4,94 trillones de Reales (cerca de 2,2 trillones de dólares). Esta ubicación privilegiada en términos del producto se sustenta en el tamaño continental de su territorio el cual contiene una variedad y abundancia de recursos naturales (biodiversidad, agua, florestas, selvas, bosques, enormes planicies, pantanal, 
etc.), energéticos (hidrocarburos, agrocombustibles, hidroelectricidad, energía solar, parques eólicos, etc.), minerales (hierro, acero, uranio, metales preciosos, etc.). Por lo mismo, la incorporación de este país al grupo de los llamados países emergentes o BRICS ${ }^{10}$ no hace más que fortalecer la imagen de que Brasil será junto a los otros miembros de los “emergentes” la gran potencia que despuntará a mediano plazo en el ámbito global. Precisamente el que Brasil sea miembros de los BRICS no garantiza que la relación con sus vecinos latinoamericanos mejore, al contrario, ello puede significar -como apuntan muchos analistas- que Brasil asuma definitivamente su posición de nación con vocación imperialista, donde el resto de países latinoamericanos pase a constituirse en la periferia de la nueva potencia.

Indudablemente, la relación de Brasil con sus vecinos de América Latina es compleja y múltiple. En algunos casos se puede apreciar un acercamiento a países que poseerían la misma sensibilidad de izquierda o progresista. Ella es ejemplar en el caso de Cuba, país con el cual se han firmado una serie de convenios importantes tales como la contratación de 30 mil médicos cubanos para participar en el programa "Mais Médicos" del Gobierno Federal. Por su parte, el tratado firmado recientemente para invertir un billón de dólares en la modernización del Puerto de Mariel beneficiará al Estado cubano, pero asimismo a las empresas brasileñas. ${ }^{11}$ Es decir, aparte de las simpatías políticas, los trabajos de reconstrucción de este puerto se han transformado en una excelente oportunidad de negocios para las grandes empresas contratistas brasileñas (en este caso Odebrecht $)^{12}$ y de igual forma para las empresas procesadoras -especialmente de productos de alta tecnología como biofármacos- se puedan instalar en esa zona económica privilegiada a escasos kilómetros de Miami y del mercado estadounidense. La proximidad con el nuevo Canal de Panamá es ciertamente un elemento a ser considerado, por la facilidad de acceder a los mercados ubicados en la región del Pacifico. En su discurso durante la inauguración de la primera fase del Convenio, la presidenta Dilma Rousseff declaró:

"Es un proceso gana-gana, Cuba gana y Brasil gana. Es un buen negocio (...) Nosotros continuaremos haciendo inversiones en el área portuaria en Brasil. Brasil es hoy un país líder en América Latina y tiene sus responsabilidades. Así como saludamos a los países desarrollados que, al hacer inversiones, financian el abastecimiento de sus empresas nacionales, Brasil también financió el Puerto de Mariel, que por ejemplo, ahora en el acuerdo, quien abastece los equipamientos, los bienes y servicios son empresas brasileñas. Más de 400 empresas brasileñas participan de este esfuerzo, generando empleo y renta”. (Blog do Planalto, 28/01/2014).

Con ese supuesto, Brasil ha tenido una política de "generosidad" con los países más pobres de la región, como es el caso del apoyo proporcionado por la Misión de Paz en Haití, donde Brasil tuvo un papel activo en la creación de la Misión de Estabilización de Naciones Unidas en Haití (MINUSTAH), que desde su inicio en 2004 se encuentra bajo el Comando Militar del ejército brasileño. La actuación de Brasil en el país caribeño 
responde de igual forma a una orientación de la política externa brasileña de tener un mayor protagonismo en los asuntos internacionales, buscando inclusive un asiento permanente en el Consejo de Seguridad de las Naciones Unidas. En realidad, Estados Unidos, Francia y Canadá dirigirían la inteligencia y la planificación estratégica de la MINUSTAH. La única novedad es que le traspasaron a Brasil el comando de las tropas que provienen en su mayoría de países de Sudamérica y de Centroamérica: Argentina, Uruguay, Brasil, Chile, Perú, Guatemala, Bolivia, Ecuador, Paraguay, El Salvador y Honduras.

¿Pero cuál ha sido el resultado concreto de estas acciones en Haití? Según entidades de derechos humanos, después de 10 años de ocupación, el país continúa con serios problemas sociales, políticos e institucionales, con una clara regresión democrática, represión violenta y sistemática de las manifestaciones populares y ataques a dirigentes de la oposición. La MINUSTAH incluso sustentaría una manipulación grosera de los procesos electorales e institucionales y la entrada libre de capitales transnacionales para controlar espacios estratégicos de la economía, incluyendo la megaminería, el turismo de lujo, las empresas que exportan productos sin pagar impuestos y la agroindustria exportadora. En ese contexto, el senado de Haití solicitó en dos ocasiones la retirada de las tropas. Investigaciones recientes indican que $89 \%$ de la populación rechaza la presencia de la MINUSTAH y la secuencia de movilizaciones masivas, que siguen aumentando desde octubre de 2013, pidiendo la renuncia del presidente, reclama sistemáticamente por el fin da ocupación.

Aún más, estas acciones de cooperación, concesiones y a veces de "renuncia” por parte de Brasil, han quedado asimismo en evidencia en los casos de Bolivia, Paraguay y Ecuador, países en los cuales Brasil tenía importantes inversiones en los sectores de hidrocarburos y energético. ${ }^{13}$

Con relación a Bolivia, el gobierno de Evo Morales decidió el año 2006 proceder a la nacionalización de sus recursos de hidrocarburos, para lo cual llegó inclusive a movilizar su ejército para realizar las tareas de recuperación de las instalaciones de las empresas refinadoras existentes en el país, entre ellas Petrobras. Sin embargo, una llamada de Marco Aurelio García -consejero para asuntos internacionales del Palacio do Planalto- al vicepresidente boliviano Álvaro García Linera, consiguió que este último desautorizara una resolución del Ministro de Energía que pretendía aplicar el decreto de nacionalización de hidrocarburos, que precisamente incluía las refinerías controladas por Petrobras. Ante dicha situación el entonces Ministro se vio -según sus propias palabras- en la obligación moral de renunciar. Posteriormente el ex Ministro denunciará en un artículo que "el interés brasileño no solo por el presente, sino también por el futuro ocasiona la susceptibilidad de quienes recuerdan que los 38 años de ‘olvido' del proyecto gas-químico está asociado con la promesa de instalar 24 termoeléctricas en territorio boliviano con parte del gas destinado a San Pablo, agravado por la exportación fraudulenta de gas, mediante asociación con Repsol y el no pago por el gas húmedo, durante los primeros ocho años 
de vigencia del contrato boliviano-brasileño. En la situación presente, Bolivia se asemeja a un enfermo muy grave que, en opinión de Lula y Marco Aurelio, podría sobrevivir con el suero por goteo que le suministraría Brasil, a condición de no desarrollarse por sí misma”. ${ }^{14}$

Esta situación solo vino a reforzar el malestar presente entre muchos bolivianos con respecto al despojo que han sufrido por parte de Brasil desde el siglo XVIII. Los orígenes de dicha expropiación histórica de la cual habría sido víctima Bolivia se remontan a fines de ese siglo, cuando Bolivia perdió, en un breve periodo de tiempo, un espacio geográfico gigantesco, de casi 300 mil km². ${ }^{15}$ Efectivamente, tal como lo expone claramente el ex presidente boliviano Carlos Mesa, desde el acuerdo que provocó la incorporación de Acre al territorio brasileño "la tesis del sub-imperialismo brasileño quedó fuertemente anclada en la interpretación boliviana de nuestras relaciones con el vecino del este". ${ }^{16}$

En otro conflicto ampliamente difundido, el gobierno de Rafael Correa decidió en octubre de 2008 expulsar a la constructora Odebrecht bajo la acusación de no cumplir con los compromisos contractuales acordados con el estado ecuatoriano. El principal motivo de esta medida drástica fue que las turbinas de la represa San Francisco sufrieron fallas técnicas de consideración un año después de haber sido instaladas, situación que llevó a la paralización de la represa, comprometiendo, de esta manera, en un porcentaje significativo la generación y el suministro de energía necesario para las actividades de dicho país. Por su parte, la empresa brasileña que declaró "lamentar los inconvenientes causados al Estado ecuatoriano", ejerció presiones sobre el gobierno de Brasil para que este intercediera ante el presidente Correa, el cual finalmente no reconsideró la medida tomada por su gobierno. En todo caso, la expulsión de la Odebrecht no liberó a Ecuador del pago de la deuda con el BNDES, lo cual significaba en concreto que este país asumió una deuda de 600 millones de dólares con el Estado brasileño para realizar un proyecto hidroeléctrico cuyos posteriores desperfectos lo transformaron en una obra inutilizable. No obstante, sintiéndose injuriado, el gobierno brasileño reaccionó con dureza frente a la decisión de Ecuador y llegó a suspender por un largo periodo todos los proyectos de cooperación e inversión destinados a ejecutar mejorías de la infraestructura que estaban siendo discutidos entre ambos países. A su vez, Ecuador amenazó con la posibilidad de nacionalizar todos los campos petroleros bajo operación de la Petrobras si dicha empresa no se adaptaba a la nueva política de hidrocarburos decretada por esa nación. En síntesis, una disputa entre el gobierno ecuatoriano y una empresa privada se transformó en un problema de estado para ambos países. En gran medida eso se debe al tipo de relación que mantiene el Estado brasileño con sus empresas y en este caso, reforzado por el estrecho vínculo existente entre la constructora Odebrecht y el Partido de los Trabajadores. ${ }^{17}$

Por cierto, algunas “concesiones” realizadas por el Estado brasileño representan solo una dimensión de esa compleja lógica integración/dominación/dependencia. Si bien, como señalábamos en líneas previas que Bra- 
sil tuvo un comportamiento tolerante ante el intento de ocupación y nacionalización de sus empresas por parte del gobierno boliviano o de la expulsión de una de sus empresas desde territorio ecuatoriano, por otra parte, el Estado brasileño no llegó en ninguna hipótesis a negociar la cesación de pagos, el fin de la cláusula indemnizatoria o la condonación de la deuda contraída por ambos gobiernos. Y desde el punto de vista de los países mencionados y del resto de los vecinos regionales, estos actos de "recuperación” se pueden ver como la culminación de un largo recorrido histórico de contención o de resistencia al expansionismo brasileño en el plano de este vínculo Estado-empresas. Precisamente, la relación estado-empresas en el contexto brasileño ha sido marcada por una especie de simbiosis entre el gobierno y las empresas, especialmente de aquellas vinculadas a la construcción civil. Dicha fusión se enmarca dentro de la perspectiva de internacionalización de las compañías brasileñas dentro de la propuesta denominada como novo desenvolvimentismo (neodesarrollismo), que representa la política de internacionalización en su segunda fase (la primera fase se inició en la década del setenta, durante la vigencia del llamado “milagro brasileño” $)^{18}$. En la etapa actual, más que agentes económicos las empresas transnacionales brasileñas han ido construyendo un espacio de diplomacia en que las empresas desempeñan un papel fundamental en la relación con los gobiernos, (gobierno-empresa) agregando un nuevo vector de articulación a las ya consagradas relaciones diádicas entre gobiernos y entre las empresas. ${ }^{19}$

A partir de estos antecedentes, algunos autores señalan que ellos demostrarían fehacientemente la vocación imperial y el papel de Brasil en el contexto regional. En ese estado de espíritu se puede inserir la afirmación plasmada en el libro de Raúl Zibechi: "La vinculación de la burguesía nacional con las cúpulas del sindicalismo y, también, con la burguesía de los países aledaños ha permitido a las empresas brasileñas extender sus dominios territoriales, consolidándolas como transnacionales que funcionan a la par con las de los imperios de antaño (...) Es decir, Brasil Potencia es posible gracias a la alianza entre un sector decisivo del movimiento sindical y del aparato estatal federal, con la burguesía brasileña y con las fuerzas armadas". ${ }^{20}$

Resulta interesante constatar que este sentimiento "anti-imperialista” presente entre muchos políticos e intelectuales de países vecinos, no ha sido percibido por el empresariado y las elites gobernantes de Brasil. Al contrario, tiende a prevalecer la idea de que Brasil es demasiado generoso y concesivo con sus vecinos menores, invocándose para ello los altos volúmenes de recursos que destina este país para financiar la construcción de grandes obras de infraestructura en prácticamente todos los países del hemisferio.

Hace algunos años atrás Ruy Mauro Marini expuso con bastante consistencia esta fuerte alianza existente a partir de los años cuarenta desde el llamado Estado Novo- entre fracciones del capital industrial y de empresas agro-exportadoras, cuando la expansión del proceso de indus- 
trialización necesitaba mantener una política de cambio desvalorizado con el objetivo de compensar el todavía insuficiente papel de los exportadores en la generación de las divisas necesarias para financiar las exigencias de importaciones de bienes de producción para la naciente industria brasileña. Esta alianza se consolido después del golpe militar de 1964 y se constituyó en una pieza relevante en la dinámica sub-imperialista emprendida por Brasil hasta mediados de los años ochenta. ${ }^{21}$

Durante el presente siglo, esta búsqueda de expansión en los mercados externos se consolida, aunque ahora con mayor énfasis en la exportación de capitales, principalmente a través de inversiones directas. Es en ese contexto en el cual surgen las políticas públicas orientas a incentivar la internacionalización de las actividades de préstamos y créditos concedidos por el BNDES hacia el resto de los países del hemisferio, inclusivo instalando filiales en varios de ellos. Pero no solo eso, la banca privada brasileña se encuentra igualmente en una acelerada fase de expansión en los países de la región y la presencia de entidades financieras como Bradesco, Itaú Unibanco, BTG Pactual, ya son parte del paisaje de muchas ciudades latinoamericanas. ${ }^{22}$

En síntesis, nos enfrentamos a un escenario contradictorio en que por una parte Brasil aparece como un gran socio dispuesto a crear y fortalecer los lazos y la integración entre todas las naciones del continente. Diferentemente, Brasil se presenta como una fuerza geopolítica tan grande con relación a sus vecinos, que "les rompe las costillas aun cuando quiere abrazarlos amistosamente”. ${ }^{23} \mathrm{O}$ incluso Brasil puede ser visualizado como una potencia con vocación imperialista que orienta toda su política exterior con la finalidad de transformarse en una fuerza avasalladora e incontrarrestable en esta zona del mundo. Se pueden encontrar argumentos para defender diversas tesis en esta relación compleja y multidimensional que se establece entre el país-continente y sus vecinos de América Latina.

Considerando estas latentes o, a veces, evidentes contradicciones y tensiones en las relaciones que establece Brasil con el resto de los países de la región, la Revista Polis invitó a todos los interesados en esta temática a enviar sus contribuciones para acompañarnos en la tarea de reflexionar sobre la trayectoria histórica de dicha relación y sus impactos sobre el futuro del continente. De esta manera, se recibieron diversos aportes y miradas involucrando a varios países -aparte de Brasil- como Argentina, Bolivia, Chile, Colombia, Perú, Uruguay y Paraguay. Estos trabajos nos permiten tener una panorámica de la producción reciente en torno a esta problemática, aportando con significativos subsidios teóricos el enorme desafío de transformar la integración regional en un efectivo estímulo al desarrollo, la autonomía y la soberanía para el conjunto de las naciones y pueblos de esta parte del planeta.

Abriendo la sección monográfica, un grupo de investigadores de la Universidad Federal del ABC, en Santo Andrés, São Paulo, se dedica a 
analizar el papel de Brasil y sus intereses en la construcción de Cachuela Esperanza, Bolivia. El trabajo plantea que la ampliación de la matriz energética brasileña ha despertado el interés de este país por desarrollar y usufructuar del gran potencial que representan las caídas y el rápido flujo de agua en los afluentes del río Madeira, en especial el río Beni, donde se planea la construcción de una hidroeléctrica en el sector de Cachuela Esperanza. Este proyecto que similarmente se inscribe en el marco de la Iniciativa para la Integración de Infraestructura Regional Sud-Americana (IIRSA), ha estado rodeado de cuestionamientos y controversias por cuanto aparte de tener un fuerte impacto ambiental sobre la calidad del agua, la devastación de especies vegetales y la migración de peces y aves, tendría aún graves consecuencias sobre las comunidades indígenas y campesinas que habitan ese territorio.

Seguidamente, Fabio Luis Barbosa dos Santos en su artículo A problemática brasiguaia e os dilemas da projeção regional brasileira analiza los meandros de la conflictiva relación entre los migrantes brasileños en territorio paraguayo, los llamados “brasiguaios”. El trabajo se inicia exponiendo el marco histórico en que se presenta la migración masiva de brasileños en dirección al país vecino, para luego proceder a analizar el impacto de la expansión del cultivo de la soja en territorio paraguayo por parte de empresas transnacionales y con participación activa de los brasiguaios, cuestión que ha generado un aumento de los conflictos en el campo y la subsecuente tensión de relaciones entre los gobiernos de Brasil y Paraguay, especialmente porque estos agentes del agro-negocio se mostraron como un importante factor de desestabilización del gobierno democrático de Fernando Lugo, contrariando la estrategia de la política exterior brasileña para la región.

En A revitalização do Mercosul no contexto da diplomacia de Lula para a América do Sul, Raquel Paz dos Santos nos entrega un estudio de la política exterior orientada específicamente para consolidar el Mercosur durante el primer gobierno de Lula, entre 2003 y 2007. En el referido ensayo, la autora busca interpretar un conjunto de iniciativas asumidas por el gobierno Lula con la finalidad de expandir y consolidar los lazos de integración a nivel de los países integrantes del Mercosur, por medio de proyectos en el área de infraestructura, energía, seguridad y defensa, intentando por esta vía fortalecer la institucionalización de la alianza y posicionando a Brasil como un actor clave en dicho proceso de integración.

Un cuartoartículo de la sección de autoría de Rafael Nascimento Gomes discurre sobre las relaciones establecidas entre los gobiernos de Getulio Vargas y Gabriel Terra en la década del treinta. De esta manera, Um ensaio sul-americano: as nossas primeiras aproximações das relações entre o Brasil e o Uruguai na década de 1930, se plantea como propósito desvendar los interludios de la política exterior uruguaya que buscaba una mayor inserción y proyección en el escenario internacional a través de un estrechamiento de vínculos con Europa y Estados Unidos, pero sin descuidar sus relaciones con Brasil y con el resto de la región. 
Desde una perspectiva culturalista e histórica, el artículo de Carlos Roberto da Rosa Rangel se dedica a describir y analizar As festas como formas de integração na fronteira Brasil-Uruguai (1930-1945). La reflexión del autor se centra en mostrar como las fiestas cívicas y carnavalescas realizadas entre las ciudades fronterizas de Santana do Livramento en Brasil y Ribera en Uruguay ayudaron a forjar un sentimiento de cultura local, el cual contrastaba con la política desarrollada por estados nacionales de carácter centralizador y homogeneizador, que encarnaban los gobiernos de Getúlio Vargas en Brasil y Gabriel Terra en Uruguay. La confraternización entre estas dos ciudades separadas por una calle, representaba entonces la posibilidad de construir un espacio de transgresión colectivo y del mismo modo de formación de lazos de solidaridad y reciprocidad entre los vecinos, lo cual generó una cultura popular y festiva común, que superaba las barreras artificiales impuestas por sus respectivos gobiernos durante las décadas de 1930 y 1940.

En el ensayo de Soledad Stoessel titulado Giro a la izquierda en la América Latina del siglo XXI se realiza una extensa revisión bibliográfica en torno a dos temas relevantes que emergen en el debate de nuestras sociedades en los albores del presente siglo. En ese sentido, la idea de post-neoliberalismo y el llamado "retorno del Estado" aparecen como elementos insoslayables de diferentes interpretaciones conceptuales y análisis políticos. A partir de ambos ejes, la autora organiza y sistematiza un conjunto de estudios y ensayos que reflexionan sobre la naturaleza y papel de las izquierdas contemporáneas a partir de la tesis de "las dos izquierdas" y en segundo lugar se plantea los desafíos y estrategias que representa la superación del modelo neoliberal y el giro a la izquierda en la mayor parte de los países de América Latina, incluyendo el gigante hemisférico.

Por último, cerrando esta sección monográficaPatrícia Cabral de Vasconcellos aborda el tema de As construtoras brasileiras e o processo de integração regional na América do Sul, en el cual plantea que a pesar de que el proceso de internalización de las empresas brasileñas de ingeniería y construcción tuvo inicio en la década del setenta, es a partir de la Iniciativa para la Integración de Infraestructura Regional Sud-Americana (IIRSA) en el año 2002 que dicha expansión se presenta con mayor intensidad. En ese sentido, la presencia y consolidación de las constructoras brasileñas en América del Sur puede ser interpretada como parte de una alianza estratégica de intereses entre el Estado y estas empresas, en el cual el discurso de integración regional difundido por la diplomacia brasileña se encuentra en los hechos más asociado con el objetivo de consolidar la internacionalización de las empresas contratistas brasileñas en el ámbito regional.

En este número incluimos en la sección Cartografías para el Futuro dos excelentes artículos. El primero de Cristian Parker titulado El Mundo Académico y las Políticas Públicas frente a la urgencia del desarrollo sustentable en América Latina y el Caribe lleva a cabo un análisis, a la luz de las transformaciones de la sociedad del conocimiento y las exigencias propias de la complejidad de la ecología y el ambiente, de las interacciones 
entre el mundo de la academia y el mundo de las políticas públicas. Constata así un serio déficit en función de las tareas necesarias para el desarrollo sustentable y propone una nueva relación entre los mundos de la academia y de las políticas públicas que asuma el objetivo de la transición hacia la sustentabilidad, la integración y la cooperación regional, que genere un espacio para la democracia ambiental, lo cual requiere revisar el diseño e implementación de las políticas públicas para la gobernanza ambiental.

El segundo artículo se titula Internet y Comunidad: Una aproximación desde el enfoque del estudio de redes personales y su autor,José Ignacio Porras Martínez, se propone aportar a la renovación metodológica en el campo de la investigación social, relevando las potencialidades del uso del estudio de las redes personales a partir de la aplicación de conceptos, métodos y técnicas propios del enfoque del Análisis de Redes Sociales (ARS). Presenta los aprendizajes obtenidos a partir de la aplicación de este método en la investigación realizada para evaluar el impacto de Internet en la formación de capital individual y colectivo en un conjunto seleccionado de localidades de la Patagonia chilena caracterizadas por sus críticos niveles de aislamiento.

El número se completa con su sección Propuestas y avances de investigación que incluye un total de once artículos y en su sección Comentarios y reseñas de libros un total de cinco trabajos. 


\section{Notas}

${ }^{1}$ Afirmamos esto, sin entrar en el mérito de la discusión con respecto a lo que significa ser en la actualidad socialista, de izquierda o progresista en el mundo y en América latina, lo cual daría asunto para otro trabajo de mayor envergadura.

${ }^{2}$ Ver, por ejemplo, Rodrigo Lara Serrano, Estados Unidos pierde influencia en América Latina, AméricaEconomía, 31/10/2008; Janette Habel, ¿Washington perdió a América Latina?, Le Monde Diplomatique, 16/12/2007; Immanuel Wallerstein, La irresistible decadencia del Imperio americano, en el sitio La Haine, 24/09/2007.

${ }^{3}$ De hecho, Mercosur ha mostrado ser un acuerdo plagado de dificultades que lo comprometen como un efectivo espacio de integración. Por ejemplo, hasta ahoraha sido difícil y demorado el proceso para unificar la moneda pues ello implica tener una política de convergencia monetaria, la cual precisa de una inflación similar y controlada por los gobiernos. De lo contrario, no es posible siquiera pensar en una moneda común. A pesar de estos y otros obstáculos a la integración sub-regional, la decidida voluntad de fortalecer Mercosur ha significado que Brasil aumentará sus intercambios comerciales con los países socios en más del 200 por ciento en los últimos años.

${ }^{4}$ Bernardo Sorj y Sergio Fausto, "El papel de Brasil en América del Sur: estrategias y percepciones mutuas”, en Sorj y Fausto (comp.). Brasil y América del Sur: Miradas cruzadas, Buenos Aires: Catálogos/Plataforma Democrática, 2011.

${ }^{5}$ El contrato conla constructora brasileña OAS fue anulado en abril de 2012 luego que el gobierno boliviano postergara la decisión de construir la vía hasta después de conocerse los resultados de la consulta que se realizaría durante el curso de ese año y que serían difundidos a mediados de diciembre.

${ }^{6}$ Existe un informe elaborado en conjunto por Caritas Boliviana y la Asamblea Permanente de Derechos Humanos de ese país en el que se concluye que "la consulta del Gobierno fue precedida y acompañada por regalos, prebendas y promesas de desarrollo y servicios que condicionaron los criterios de libre y buena fe".

${ }^{7}$ El llamado caso TIPNIS representa un caso emblemático de las contradicciones existentes entre gobiernos que dicen respetar el medioambiente y las comunidades, pero que a la hora de atender a los intereses de las empresas y los productores, acaban por abdicar de su discurso ambientalista para privilegiar una pretendida vocación desarrollista que favorecería al conjunto de la población y no solamente a un segmento minoritario, como lo son los pueblos originarios y comunidades que se encuentran asentadas en los territorios en disputa.

${ }^{8}$ En todo caso, la diplomacia brasileña hasta ahora no ha conseguido el ansiado asiento permanente en dicho organismo.

${ }^{9}$ La voluntad de establecer una política de mayor acercamiento con América Latina y los países africanos de lengua portuguesa (Angola, Mozambique, Cabo Verde, Guinea Bissau, San Tomé y Príncipe y Timor-Este, se encuentra claramente retratada en la creación, durante el gobierno Lula, de dos universidades orientadas a alumnos provenientes de la región latinoamericana y de los países africanos lusófonos. Son ellas la Universidad Federal de la Integración Latino-Americana (UNILA) y la Universidad de 
Integración Internacional de la Lusofonía Afro-Brasilera (UNILAB).

${ }^{10}$ Los BRICS son: Brasil, Rusia, India, China y Sudáfrica. Algunos economistas han sugerido además la incorporación al grupo de Corea del Sur.

${ }^{11}$ Las obras del puerto están siendo financiadas por el BNDES, siendo que del presupuesto inicial de US\$ 957 millones, el equivalente a unos US\$ 800 millones se han destinado a la contratación de servicios y equipamientos producidos en Brasil, lo cual ha generado cerca de 150 mil empleos en este país. El puerto responde por la mayor cantidad del presupuesto invertido por Brasil en Cuba, que en total alcanza la suma de 1,6 billones de dólares.

${ }^{12}$ Para realizar estas grandes obras, muchos gobiernos han aceptado el apoyo de Brasil, que por medio del BNDES ha efectuado préstamos anuales por un monto de 80 mil millones de dólares. En general la condición impuesta a los países financiados, es que las empresas constructoras sean brasileñas. Junto a la mencionada Odebrecht, se destacan en la construcción civil empresas tales como OAS, Andrade Gutiérrez, Mendes Júnior, Queiroz Galvão o Camargo Corrêa.

${ }^{13}$ La ocupación de una refinaría de la Petrobras en Bolivia por parte del gobierno de ese país, fue considerada una afrenta por muchos políticos y especialistas en relaciones internacionales, quienes presionaron al gobierno a tomar medidas vehementes para recuperar lo que consideraban un patrimonio de toda la nación brasileña.

${ }^{14}$ Andrés Soliz Rada, Arremetida brasileña, en sitio Rebelión, 26/04/2010.

${ }^{15}$ Bolivia cedió más de 100 mil km² en la región del río Madera en el Tratado que se firmó en 1867 entre el Presidente Mariano Melgarejo de Bolivia y el Emperador Pedro II de Brasil. Posteriormente, ante la inminencia de un conflicto bélico total por la posesión del territorio de Acre, Bolivia aceptó ceder prácticamente todo ese territorio de 190 mil km² a cambio de una compensación pecuniario. Ambos acuerdos le restaron a Bolivia el equivalente a más del $25 \%$ de su actual territorio.

${ }^{16}$ Carlos Mesa, "Bolivia y Brasil: los meandros del camino”, en Sorj y Fausto (comp.). Brasil y América del Sur: Miradas cruzadas, Buenos Aires: Catálogos/Plataforma Democrática, 2011.

${ }^{17}$ Analistas calculan que los aportes y donaciones de campaña que beneficiaron al PT y sus candidatos en las elecciones de 2010, tanto por parte de la Odebrecht como de otras empresas constructoras, alcanzó la cifra de 15 millones de dólares. Ver Raúl Zibechi, Brasil Potencia, México: Bajo Tierra Ediciones, 2012, p. 48.

${ }^{18}$ Como lo destaca una especialista: "la internacionalización se hace necesaria y la diplomacia responde a las demandas del desarrollo nacional no solamente desde la óptica de sus agentes de Estado, sino que también, en la actuación de sus agentes de mercado, nacional e internacionalmente. La integración de la infraestructura y del mercado regional es una condición indispensable para el alcance de los objetivos del nuevo desarrollismo". Patrícia Vasconcellos, O papel das empresas transnacionais brasileiras de engenharia e construção na integração sul-americana. En: Brasil e América Latina. Percursos e Dilemas de uma Integração, Fortaleza: Ediciones UFC, 2014, pp. 77-113. 
Polis, Revista Latinoamericana, Volumen 13, $N^{\circ}$ 38, 2014

19 "En la diplomacia Estado-empresa, las empresas son capaces de influenciar en las negociaciones y tomas de decisiones en aquellos asuntos que antes eran vistos como un campo específico de las relaciones entre Estados. La relación Estado-empresa se transforma, para ambos lados, en una estrategia política para el desarrollo económico global”.Vasconcellos, op. cit., p. 105.

${ }^{20}$ Zibechi, op. cit., pp. 10-12.

${ }^{21}$ Ruy Mauro Marini, América Latina: integración e dependencia, São Paulo: Brasil Urgente, 1992.

${ }^{22}$ El Banco do Brasil que posee una importante actuación en el hemisferio, es una institución estatal, aunque constituida como una sociedad de economía mixta.

${ }^{23}$ Andrés Soliz Rada, Geopolítica brasileña, en sitio Rebelión, 27/09/2011. 